\title{
Fejér and Hermite-Hadamard Type Inequalities for Harmonically Convex Functions
}

\author{
Feixiang Chen ${ }^{1}$ and Shanhe $\mathrm{Wu}^{2}$ \\ ${ }^{1}$ School of Mathematics and Statistics, Chongqing Three Gorges University, Wanzhou, Chongqing 404000, China \\ ${ }^{2}$ Department of Mathematics and Computer Science, Longyan University, Longyan, Fujian 364012, China
}

Correspondence should be addressed to Shanhe Wu; shanhewu@gmail.com

Received 21 June 2014; Accepted 23 July 2014; Published 6 August 2014

Academic Editor: Yu-Ming Chu

Copyright (c) 2014 F. Chen and S. Wu. This is an open access article distributed under the Creative Commons Attribution License, which permits unrestricted use, distribution, and reproduction in any medium, provided the original work is properly cited.

We establish a Fejér type inequality for harmonically convex functions. Our results are the generalizations of some known results. Moreover, some properties of the mappings in connection with Hermite-Hadamard and Fejér type inequalities for harmonically convex functions are also considered.

\section{Introduction}

Let $f: I \subseteq \mathbb{R} \rightarrow \mathbb{R}$ be a convex function and $a, b \in I$ with $a<b$; then

$$
f\left(\frac{a+b}{2}\right) \leq \frac{1}{b-a} \int_{a}^{b} f(t) d t \leq \frac{f(a)+f(b)}{2} .
$$

Inequality (1) is known in the literature as the HermiteHadamard inequality. Fejér [1] established the following weighted generalization of inequality (1).

Theorem 1. If $f:[a, b] \rightarrow \mathbb{R}$ is a convex function, then the following inequality holds:

$$
\begin{aligned}
f\left(\frac{a+b}{2}\right) \int_{a}^{b} p(x) d x & \leq \int_{a}^{b} f(x) p(x) d x \\
& \leq \frac{f(a)+f(b)}{2} \int_{a}^{b} p(x) d x,
\end{aligned}
$$

where $p:[a, b] \rightarrow \mathbb{R}$ is positive, integrable, and symmetric with respect to $x=(a+b) / 2$.

Some generalizations, refinements, variations, and improvements of inequalities (1) and (2) were investigated by $\mathrm{Wu}$ [2], Chen and Liu [3], Sarikaya and Ogunmez [4], and Xiao et al. [5], respectively.
In [6], Dragomir proposed an interesting HermiteHadamard type inequality which refines the left hand side of inequality of (1) as follows.

Theorem 2 (see [6]). Let $f$ be a convex function defined on $[a, b]$. Then $H$ is convex, increasing on $[0,1]$, and for all $t \in$ $[0,1]$, one has

$$
f\left(\frac{a+b}{2}\right)=H(0) \leq H(t) \leq H(1)=\frac{1}{b-a} \int_{a}^{b} f(x) d x,
$$

where

$$
H(t)=\frac{1}{b-a} \int_{a}^{b} f\left(t x+(1-t) \frac{a+b}{2}\right) d x .
$$

An analogous result for convex functions which refines the right hand side of inequality (1) was obtained by Yang and Hong in [7] as follows.

Theorem 3 (see [7]). Let $f$ be a convex function defined on $[a, b]$. Then $F$ is convex, increasing on $[0,1]$, and for all $t \in$ $[0,1]$, one has

$$
\frac{1}{b-a} \int_{a}^{b} f(x) d x=F(0) \leq F(t) \leq F(1)=\frac{f(a)+f(b)}{2},
$$


where

$$
\begin{aligned}
F(t)=\frac{1}{2(b-a)} \int_{a}^{b}[ & f\left(\left(\frac{1+t}{2}\right) a+\left(\frac{1-t}{2}\right) x\right) \\
+ & \left.f\left(\left(\frac{1+t}{2}\right) b+\left(\frac{1-t}{2}\right) x\right)\right] d x
\end{aligned}
$$

Yang and Tseng in [8] established the following Fejér type inequalities, which is the generalization of inequalities (3) and (5) as well as the refinement of the Fejér inequality (2).

Theorem 4 (see [8]). If $f$ is convex on $[a, b], p:[a, b] \rightarrow \mathbb{R}$ is positive, integrable, and symmetric about $x=(a+b) / 2$. Then $P$ and $Q$ are convex, increasing on $[0,1]$, and for all $t \in[0,1]$, one has

$$
\begin{aligned}
f\left(\frac{a+b}{2}\right) \int_{a}^{b} p(x) d x & =P(0) \leq P(t) \leq P(1) \\
& =\int_{a}^{b} f(x) p(x) d x \\
& =Q(0) \leq Q(t) \leq Q(1) \\
& =\frac{f(a)+f(b)}{2} \int_{a}^{b} p(x) d x
\end{aligned}
$$

where

$$
\begin{aligned}
& P(t)= \int_{a}^{b} f\left(t x+(1-t) \frac{a+b}{2}\right) p(x) d x \\
& Q(t)=\frac{1}{2} \int_{a}^{b}\left[f\left(\left(\frac{1+t}{2}\right) a+\left(\frac{1-t}{2}\right) x\right) p\left(\frac{x+a}{2}\right)\right. \\
&\left.\quad+f\left(\left(\frac{1+t}{2}\right) b+\left(\frac{1-t}{2}\right) x\right) p\left(\frac{x+b}{2}\right)\right] d x
\end{aligned}
$$

In $[9,10]$, İşcan and Wu gave the definition of harmonic convexity as follows.

Definition 5. Let $I \subseteq \mathbb{R} \backslash\{0\}$ be a real interval. A function $f: I \rightarrow \mathbb{R}$ is said to be harmonically convex if

$$
f\left(\frac{x y}{t x+(1-t) y}\right) \leq t f(y)+(1-t) f(x),
$$

for all $x, y \in I$ and $t \in[0,1]$. If the inequality in (10) is reversed, then $f$ is said to be harmonically concave.

The following Hermite-Hadamard inequality for harmonically convex functions holds true.

Theorem 6 (see [9]). Let $f: I \subseteq \mathbb{R} \backslash\{0\} \rightarrow \mathbb{R}$ be $a$ harmonically convex function and $a, b \in I$ with $a<b$. If $f \in L(a, b)$, then one has

$$
f\left(\frac{2 a b}{a+b}\right) \leq \frac{a b}{b-a} \int_{a}^{b} \frac{f(x)}{x^{2}} d x \leq \frac{f(a)+f(b)}{2} .
$$

In [10], İşcan and Wu established the following HermiteHadamard inequalities for harmonically convex functions via the Riemann-Liouville fractional integral.

Theorem 7 (see [10]). Let $f: I \subseteq(0, \infty) \rightarrow \mathbb{R}$ be a function such that $f \in L(a, b)$, where $a, b \in I$ with $a<b$. If $f$ is a harmonically convex function on $[a, b]$, then the following inequalities for fractional integrals hold:

$$
\begin{aligned}
f\left(\frac{2 a b}{a+b}\right) \leq & \frac{\Gamma(\alpha+1)}{2}\left(\frac{a b}{b-a}\right)^{\alpha}\left[J_{1 / a^{-}}^{\alpha}(f \circ g)\left(\frac{1}{b}\right)\right. \\
& \left.+J_{1 / b^{+}}^{\alpha}(f \circ g)\left(\frac{1}{a}\right)\right] \\
& \leq \frac{f(a)+f(b)}{2},
\end{aligned}
$$

where $\alpha>0$ and $g(x)=1 / x$

The Riemann-Liouville fractional integrals $J_{a^{+}}^{\alpha} f$ and $J_{b^{-}}^{\alpha} f$ of order $\alpha>0$ with $a \geq 0$ are defined by

$$
\begin{aligned}
& J_{a^{+}}^{\alpha} f(x)=\frac{1}{\Gamma(\alpha)} \int_{a}^{x}(x-t)^{\alpha-1} f(t) d t, \quad x>a, \\
& J_{b^{-}}^{\alpha} f(x)=\frac{1}{\Gamma(\alpha)} \int_{x}^{b}(t-x)^{\alpha-1} f(t) d t, \quad x<b,
\end{aligned}
$$

where $\Gamma(\alpha)$ is the Gamma function defined by $\Gamma(\alpha)=$ $\int_{0}^{\infty} e^{-t} t^{\alpha-1} d t$

In this paper, we establish a Fejér type inequality for harmonically convex functions; our main result includes, as special cases, the inequalities given by Theorems 6 and 7 . Moreover, we investigate some properties of the mappings in connection to Hermite-Hadamard and Fejér type inequalities for harmonically convex functions.

\section{Fejér Type Inequality for Harmonically Convex Functions}

The following Fejér inequality for harmonically convex functions holds true.

Theorem 8. Let $f: I \subseteq \mathbb{R} \backslash\{0\} \rightarrow \mathbb{R}$ be a harmonically convex function and $a, b \in I$ with $a<b$. If $f \in L(a, b)$, then one has

$$
\begin{aligned}
f\left(\frac{2 a b}{a+b}\right) \int_{a}^{b} \frac{p(x)}{x^{2}} d x & \leq \int_{a}^{b} \frac{f(x)}{x^{2}} p(x) d x \\
& \leq \frac{f(a)+f(b)}{2} \int_{a}^{b} \frac{p(x)}{x^{2}} d x
\end{aligned}
$$

where $p:[a, b] \rightarrow \mathbb{R}$ is nonnegative and integrable and satisfies

$$
p\left(\frac{a b}{x}\right)=p\left(\frac{a b}{a+b-x}\right) .
$$


Proof. Since $f$ is a harmonically convex function on $[a, b]$, we have, for all $x, y \in[a, b]$,

$$
f\left(\frac{2 x y}{x+y}\right) \leq \frac{f(y)+f(x)}{2} .
$$

Choosing $x=a b /(t b+(1-t) a)$ and $y=a b /(t a+(1-t) b)$, we have

$$
\begin{aligned}
& f\left(\frac{2 a b}{a+b}\right) \\
& \quad \leq \frac{f(a b /(t b+(1-t) a))+f(a b /(t a+(1-t) b))}{2} \\
& \quad \leq \frac{f(a)+f(b)}{2} .
\end{aligned}
$$

Since $p$ is nonnegative and satisfies the condition of (15), we obtain

$$
\begin{aligned}
& f\left(\frac{2 a b}{a+b}\right) p\left(\frac{a b}{t b+(1-t) a}\right) \\
& \leq\left(f\left(\frac{a b}{t b+(1-t) a}\right) p\left(\frac{a b}{t b+(1-t) a}\right)\right. \\
& \left.\quad+f\left(\frac{a b}{t a+(1-t) b}\right) p\left(\frac{a b}{t a+(1-t) b}\right)\right) \times 2^{-1} \\
& \leq \frac{f(a)+f(b)}{2} p\left(\frac{a b}{t b+(1-t) a}\right) .
\end{aligned}
$$

Integrating both sides of the above inequalities with respect to $t$ over $[0,1]$, we obtain

$$
\begin{aligned}
& f\left(\frac{2 a b}{a+b}\right) \int_{0}^{1} p\left(\frac{a b}{t b+(1-t) a}\right) d t \\
& \leq \int_{0}^{1}\left(\left(f\left(\frac{a b}{t b+(1-t) a}\right) p\left(\frac{a b}{t b+(1-t) a}\right)\right.\right. \\
& \left.\left.\quad+f\left(\frac{a b}{t a+(1-t) b}\right) p\left(\frac{a b}{t a+(1-t) b}\right)\right) \times 2^{-1}\right) d t \\
& \leq \frac{f(a)+f(b)}{2} \int_{0}^{1} p\left(\frac{a b}{t b+(1-t) a}\right) d t .
\end{aligned}
$$

The proof of Theorem 8 is completed.

Remark 9. Putting $p(x) \equiv 1$ in Theorem 8 , we obtain inequality (11).

\section{Remark 10. Choosing}

$$
\begin{array}{r}
p(x)=\frac{\alpha}{2}\left(\frac{a b}{b-a}\right)^{\alpha}\left\{\left(\frac{1}{x}-\frac{1}{b}\right)^{\alpha-1}+\left(\frac{1}{a}-\frac{1}{x}\right)^{\alpha-1}\right\}, \\
(\alpha>0,0<a<b),
\end{array}
$$

in Theorem 8 , it is easy to observe that $p(a b / x)=p(a b /(a+$ $b-x)$ ).
Since

$$
\begin{aligned}
\int_{a}^{b} & \frac{p(x)}{x^{2}} d x \\
& =\int_{a}^{b} \frac{p(x)}{x^{2}} d x \\
& =\frac{\alpha}{2}\left(\frac{a b}{b-a}\right)^{\alpha} \int_{a}^{b} \frac{1}{x^{2}}\left\{\left(\frac{1}{x}-\frac{1}{b}\right)^{\alpha-1}+\left(\frac{1}{a}-\frac{1}{x}\right)^{\alpha-1}\right\} d x \\
& =\frac{\alpha}{2}\left(\frac{a b}{b-a}\right)^{\alpha} \int_{a}^{b} \frac{1}{x^{2}}\left\{\left(\frac{1}{x}-\frac{1}{b}\right)^{\alpha-1}+\left(\frac{1}{a}-\frac{1}{x}\right)^{\alpha-1}\right\} d x \\
& =\frac{\alpha}{2}\left(\frac{a b}{b-a}\right)^{\alpha} \int_{1 / b}^{1 / a}\left\{\left(u-\frac{1}{b}\right)^{\alpha-1}+\left(\frac{1}{a}-u\right)^{\alpha-1}\right\} d u \\
& =1,
\end{aligned}
$$$$
\begin{aligned}
\int_{a}^{b} & \frac{f(x)}{x^{2}} p(x) d x \\
= & \int_{a}^{b} \frac{f(x) p(x)}{x^{2}} d x \\
= & \frac{\alpha}{2}\left(\frac{a b}{b-a}\right)^{\alpha} \\
& \times \int_{a}^{b} \frac{f(x)}{x^{2}}\left\{\left(\frac{1}{x}-\frac{1}{b}\right)^{\alpha-1}+\left(\frac{1}{a}-\frac{1}{x}\right)^{\alpha-1}\right\} d x
\end{aligned}
$$$$
=\frac{\alpha}{2}\left(\frac{a b}{b-a}\right)^{\alpha}
$$$$
\times \int_{1 / b}^{1 / a} f\left(\frac{1}{u}\right)\left\{\left(u-\frac{1}{b}\right)^{\alpha-1}+\left(\frac{1}{a}-u\right)^{\alpha-1}\right\} d u
$$$$
=\frac{\alpha}{2}\left(\frac{a b}{b-a}\right)^{\alpha}\left\{\int_{1 / b}^{1 / a} f\left(\frac{1}{u}\right)\left(u-\frac{1}{b}\right)^{\alpha-1} d u\right.
$$$$
\left.+\int_{1 / b}^{1 / a} f\left(\frac{1}{u}\right)\left(\frac{1}{a}-u\right)^{\alpha-1} d u\right\}
$$$$
=\frac{\alpha}{2}\left(\frac{a b}{b-a}\right)^{\alpha}\left\{\int_{1 / b}^{1 / a} f \circ g(u)\left(u-\frac{1}{b}\right)^{\alpha-1} d u\right.
$$$$
\left.+\int_{1 / b}^{1 / a} f \circ g(u)\left(\frac{1}{a}-u\right)^{\alpha-1} d u\right\}
$$

$$
\begin{aligned}
= & \frac{\alpha}{2}\left(\frac{a b}{b-a}\right)^{\alpha} \\
& \times\left\{\Gamma(\alpha)\left[J_{1 / a^{-}}^{\alpha}(f \circ g)\left(\frac{1}{b}\right)+J_{1 / b^{+}}^{\alpha}(f \circ g)\left(\frac{1}{a}\right)\right]\right\} \\
= & \frac{\Gamma(\alpha+1)}{2}\left(\frac{a b}{b-a}\right)^{\alpha} \\
& \times\left[J_{1 / a^{-}}^{\alpha}(f \circ g)\left(\frac{1}{b}\right)+J_{1 / b^{+}}^{\alpha}(f \circ g)\left(\frac{1}{a}\right)\right],
\end{aligned}
$$


where $g(x)=1 / x$, which implies that inequality (14) can be transformed to inequality (12) under an appropriate selection of $p(x)$.

Remark 11. In Theorem 8 , taking $p(a b / x)=\omega(x)$, where $0<$ $a<b, \omega(x)$ is nonnegative, integrable, and symmetric with respect to $x=(a+b) / 2$. Then inequality (14) becomes

$$
\begin{aligned}
f\left(\frac{2 a b}{a+b}\right) \int_{a}^{b} \omega(x) d x & \leq \int_{a}^{b} f\left(\frac{a b}{x}\right) \omega(x) d x \\
& \leq \frac{f(a)+f(b)}{2} \int_{a}^{b} \omega(x) d x
\end{aligned}
$$

\section{Some Mappings in connection with Hermite-Hadamard and Fejér Inequalities for Harmonically Convex Functions}

Lemma 12. Let $f: I \subseteq \mathbb{R} \backslash\{0\} \rightarrow \mathbb{R}$ be a harmonically convex function and $a, b \in I$ with $a<b$, and let

$$
h(t)=\frac{1}{2} f\left(\frac{2 a b}{a+b-t}\right)+\frac{1}{2} f\left(\frac{2 a b}{a+b+t}\right),
$$

$t \in[0, b-a]$. Then $h$ is convex, increasing on $[0, b-a]$, and for all $t \in[0, b-a]$,

$$
f\left(\frac{2 a b}{a+b}\right) \leq h(t) \leq \frac{f(a)+f(b)}{2} .
$$

Proof. Firstly, for $x, y \in[0, b-a]$, we have

$$
\begin{aligned}
h(t x & +(1-t) y) \\
= & \frac{1}{2} f\left(\frac{2 a b}{a+b-[t x+(1-t) y]}\right) \\
& +\frac{1}{2} f\left(\frac{2 a b}{a+b+[t x+(1-t) y]}\right) \\
= & \frac{1}{2} f\left(\frac{2 a b}{t(a+b-x)+(1-t)(a+b-y)}\right) \\
& +\frac{1}{2} f\left(\frac{2 a b}{t(a+b+x)+(1-t)(a+b+y)}\right) \\
\leq & \frac{t}{2} f\left(\frac{2 a b}{a+b-x}\right)+\frac{1-t}{2} f\left(\frac{2 a b}{a+b-y}\right) \\
& +\frac{t}{2} f\left(\frac{2 a b}{a+b+x}\right)+\frac{1-t}{2} f\left(\frac{2 a b}{a+b+y}\right) \\
= & t h(x)+(1-t) h(y),
\end{aligned}
$$

and hence $h$ is convex on $[0, b-a]$.
Next, if $t \in[0, b-a]$, it follows from the harmonic convexity of $f$ that

$$
\begin{aligned}
h(t) & =\frac{1}{2} f\left(\frac{2 a b}{a+b-t}\right)+\frac{1}{2} f\left(\frac{2 a b}{a+b+t}\right) \\
& \geq f\left(\frac{2 a b}{(1 / 2)(a+b-t)+(1 / 2)(a+b+t)}\right) \\
& =f\left(\frac{2 a b}{a+b}\right) .
\end{aligned}
$$

It is easy to observe that

$$
\begin{aligned}
h(t)= & \frac{1}{2} f\left(\frac{2 a b}{a+b-t}\right)+\frac{1}{2} f\left(\frac{2 a b}{a+b+t}\right) \\
= & \frac{1}{2} f\left(2 a b \times\left(\frac{b-a+t}{b-a} a+\frac{b-a-t}{b-a} b\right)^{-1}\right) \\
& +\frac{1}{2} f\left(2 a b \times\left(\frac{b-a-t}{b-a} a+\frac{b-a+t}{b-a} b\right)^{-1}\right) \\
\leq & \frac{1}{2} \frac{(b-a)+t}{2(b-a)} f(b)+\frac{1}{2} \frac{(b-a)-t}{2(b-a)} f(a) \\
& +\frac{1}{2} \frac{(b-a)+t}{2(b-a)} f(a)+\frac{1}{2} \frac{(b-a)-t}{2(b-a)} f(b) \\
= & \frac{f(a)+f(b)}{2} .
\end{aligned}
$$

Thus inequality (24) holds.

Finally, for $0<t_{1}<t_{2} \leq b-a$, since $h$ is convex, it follows from (24) that

$$
\begin{aligned}
\frac{h\left(t_{2}\right)-h\left(t_{1}\right)}{t_{2}-t_{1}} & \geq \frac{h\left(t_{1}\right)-h(0)}{t_{1}-0} \\
& =\frac{h\left(t_{1}\right)-f(2 a b /(a+b))}{t_{1}} \geq 0,
\end{aligned}
$$

and hence, $h\left(t_{2}\right) \geq h\left(t_{1}\right)$, which means that $h$ is increasing on $[0, b-a]$. This completes the proof of Lemma 12 .

Theorem 13. Let $f: I \subseteq \mathbb{R} \backslash\{0\} \rightarrow \mathbb{R}$ be a harmonically convex function and $a, b \in I$ with $a<b$. If $f \in L(a, b)$ and $H$ is defined by

$$
\begin{aligned}
H(t)= & \frac{1}{2(b-a)} \int_{0}^{b-a} f\left(\frac{2 a b}{a+b-t x}\right) d x \\
& +\frac{1}{2(b-a)} \int_{0}^{b-a} f\left(\frac{2 a b}{a+b+t x}\right) d x \\
= & \frac{1}{b-a} \int_{a}^{b} f\left(\frac{a b}{(1-t)((a+b) / 2)+t x}\right) d x,
\end{aligned}
$$

then $H$ is convex and increasing on $[0,1]$, and

$$
\begin{aligned}
f\left(\frac{2 a b}{a+b}\right) & =H(0) \leq H(t) \leq H(1) \\
& =\frac{a b}{b-a} \int_{a}^{b} \frac{f(x)}{x^{2}} d x .
\end{aligned}
$$


Proof. It follows from Lemma 12 that

$$
h(t)=\frac{1}{2} f\left(\frac{2 a b}{a+b-t}\right)+\frac{1}{2} f\left(\frac{2 a b}{a+b+t}\right)
$$

is convex and increasing on $[0, b-a]$. Hence $H(t)$ is convex and increasing on $[0,1]$. Further, inequality (30) can be deduced from (24). Theorem 13 is proved.

Theorem 14. Let $f: I \subseteq \mathbb{R} \backslash\{0\} \rightarrow \mathbb{R}$ be a harmonically convex function and $a, b \in I$ with $a<b$. If $f \in L(a, b)$ and $G$ is defined by

$$
\begin{aligned}
G(t)= & \frac{1}{2(b-a)} \int_{0}^{b-a} f\left(\frac{2 a b}{2 a+(1-t) x}\right) d x \\
& +\frac{1}{2(b-a)} \int_{0}^{b-a} f\left(\frac{2 a b}{2 b-(1-t) x}\right) d x \\
= & \frac{1}{2(b-a)} \int_{a}^{b} f\left(\frac{2 a b}{(1+t) a+(1-t) x}\right) d x \\
& +\frac{1}{2(b-a)} \int_{a}^{b} f\left(\frac{2 a b}{(1+t) b+(1-t) x}\right) d x,
\end{aligned}
$$

then $G$ is convex and increasing on $[0,1]$, and

$$
\begin{aligned}
\frac{a b}{b-a} \int_{a}^{b} \frac{f(x)}{x^{2}} d x & =G(0) \leq G(t) \leq G(1) \\
& =\frac{f(a)+f(b)}{2} .
\end{aligned}
$$

Proof. We note that if $f$ is convex and $g$ is linear, then the composition $f \circ g$ is convex. It follows from Lemma 12 that

$$
h(t)=\frac{1}{2} f\left(\frac{2 a b}{a+b-t}\right)+\frac{1}{2} f\left(\frac{2 a b}{a+b+t}\right),
$$

and $k(t)=b-a-(1-t) x$ are increasing on $[0, b-a]$ and $[0,1]$, respectively. Hence,

$$
h(k(t))=f\left(\frac{2 a b}{2 a+(1-t) x}\right)+f\left(\frac{2 a b}{2 b-(1-t) x}\right)
$$

is convex and increasing on $[0,1]$. We infer that $G$ is convex and increasing on $[0,1]$. Furthermore, inequality (33) follows directly from (24). The proof of Theorem 14 is completed.

Theorem 15. Let $f: I \subseteq \mathbb{R} \backslash\{0\} \rightarrow \mathbb{R}$ be a harmonically convex function and $a, b \in I$ with $a<b$. If $f \in L(a, b)$ and $P$ is defined by

$$
\begin{aligned}
P(t)= & \frac{1}{2 a b} \int_{0}^{b-a} f\left(\frac{2 a b}{a+b-t x}\right) p\left(\frac{2 a b}{b+a-x}\right) d x \\
& +\frac{1}{2 a b} \int_{0}^{b-a} f\left(\frac{2 a b}{a+b+t x}\right) p\left(\frac{2 a b}{b+a+x}\right) d x \\
= & \frac{1}{a b} \int_{a}^{b} f\left(\frac{a b}{((a+b) / 2)(1-t)+t x}\right) p\left(\frac{a b}{x}\right) d x
\end{aligned}
$$

where $p:[a, b] \rightarrow \mathbb{R}$ is nonnegative and integrable and satisfies the condition of (15), then $P$ is convex and increasing on $[0,1]$, and

$$
\begin{aligned}
f\left(\frac{2 a b}{a+b}\right) \int_{a}^{b} \frac{p(x)}{x^{2}} d x & =P(0) \leq P(t) \leq P(1) \\
& =\int_{a}^{b} \frac{f(x)}{x^{2}} p(x) d x
\end{aligned}
$$

Proof. From Lemma 12 we obtain that

$$
h(t)=\frac{1}{2} f\left(\frac{2 a b}{a+b-t}\right)+\frac{1}{2} f\left(\frac{2 a b}{a+b+t}\right)
$$

is convex and increasing on $[0, b-a]$. Since $p(2 a b /(a+b+x))$ is nonnegative and satisfies $p(2 a b /(a+b+x))=p(2 a b /(a+$ $b-x)$ ), it follows that $P(t)$ is convex and increasing on $[0,1]$, while inequality (37) can be deduced from (24). Theorem 15 is proved.

Theorem 16. Let $f: I \subseteq \mathbb{R} \backslash\{0\} \rightarrow \mathbb{R}$ be a harmonically convex function and $a, b \in I$ with $a<b$. If $f \in L(a, b)$ and $Q$ is defined by

$$
\begin{aligned}
Q(t)= & \frac{1}{2 a b} \int_{0}^{b-a} f\left(\frac{2 a b}{2 a+(1-t) x}\right) p\left(\frac{2 a b}{2 a+x}\right) d x \\
& +\frac{1}{2 a b} \int_{0}^{b-a} f\left(\frac{2 a b}{2 b-(1-t) x}\right) p\left(\frac{2 a b}{2 b-x}\right) d x \\
= & \frac{1}{2 a b} \int_{a}^{b} f\left(\frac{2 a b}{(1+t) a+(1-t) x}\right) p\left(\frac{2 a b}{x+a}\right) d x \\
& +\frac{1}{2 a b} \int_{a}^{b} f\left(\frac{2 a b}{(1+t) b+(1-t) x}\right) p\left(\frac{2 a b}{x+b}\right) d x
\end{aligned}
$$

where $p:[a, b] \rightarrow \mathbb{R}$ is nonnegative and integrable and satisfies the condition of (15), then $Q$ is convex and increasing on $[0,1]$, and

$$
\begin{aligned}
\int_{a}^{b} \frac{f(x)}{x^{2}} p(x) d x & =Q(0) \leq Q(t) \leq Q(1) \\
& =\frac{f(a)+f(b)}{2} \int_{a}^{b} \frac{p(x)}{x^{2}} d x
\end{aligned}
$$

Proof. By using the same method as in the proof of Theorem 14, we obtain from Lemma 12 that

$$
h(k(t))=f\left(\frac{2 a b}{2 a+(1-t) x}\right)+f\left(\frac{2 a b}{2 b-(1-t) x}\right)
$$

is convex and increasing on $[0,1]$. Since $p(2 a b /(2 a+x))$ is nonnegative and satisfies $p(2 a b /(2 a+x))=p(2 a b /(2 b-x))$, 
we deduce that $Q(t)$ is convex and increasing on $[0,1]$. Inequality (40) follows from (24) and the identity

$$
\begin{aligned}
\frac{1}{2 a b} \int_{0}^{b-a} p\left(\frac{2 a b}{2 a+x}\right) d x= & \frac{1}{2 a b} \int_{0}^{b-a} p\left(\frac{2 a b}{2 b-x}\right) d x \\
= & \frac{1}{2}\left\{\frac{1}{2 a b} \int_{0}^{b-a} p\left(\frac{2 a b}{2 a+x}\right) d x\right. \\
& \left.+\frac{1}{2 a b} \int_{0}^{b-a} p\left(\frac{2 a b}{2 b-x}\right) d x\right\} \\
= & \frac{1}{2} \int_{a}^{b} \frac{p(x)}{x^{2}} d x .
\end{aligned}
$$

This completes the proof of Theorem 16.

Remark 17. If we put

$$
p(x)=\frac{\alpha}{2}\left(\frac{a b}{b-a}\right)^{\alpha}\left\{\left(\frac{1}{x}-\frac{1}{b}\right)^{\alpha-1}+\left(\frac{1}{a}-\frac{1}{x}\right)^{\alpha-1}\right\},
$$

in inequalities (37) and (40), respectively, we obtain the refined versions of inequality (12).

\section{Conflict of Interests}

The authors declare that there is no conflict of interests regarding the publication of this paper.

\section{Acknowledgments}

The present investigation was supported, in part, by the Youth Project of Chongqing Three Gorges University of China (no. 13QN11) and, in part, by the Foundation of Scientific Research Project of Fujian Province Education Department of China (no. JK2012049).

\section{References}

[1] L. Fejér, "Über die Fourierreihen, II," Math.Naturwiss. Anz Ungar. Akad. Wiss, vol. 24, pp. 369-390, 1906 (Hungarian).

[2] S. Wu, "On the weighted generalization of the HermiteHadamard inequality and its applications," Rocky Mountain Journal of Mathematics, vol. 39, no. 5, pp. 1741-1749, 2009.

[3] F. X. Chen and X. F. Liu, "Refinements on the HermiteHadamard inequalities for $r$-convex functions," Journal of Applied Mathematics, vol. 2013, Article ID 978493, 5 pages, 2013.

[4] M. Z. Sarikaya and H. Ogunmez, "On new inequalities via Riemann-Liouville fractional integration," Abstract and Applied Analysis, vol. 2012, Article ID 428983, 10 pages, 2012.

[5] Z. G. Xiao, Z. H. Zhang, and Y. D. Wu, "On weighted HermiteHadamard inequalities," Applied Mathematics and Computation, vol. 218, no. 3, pp. 1147-1152, 2011.

[6] S. S. Dragomir, "Two mappings in connection to Hadamard's inequalities," Journal of Mathematical Analysis and Applications, vol. 167, no. 1, pp. 49-56, 1992.
[7] G. S. Yang and M. C. Hong, "A note on Hadamard's inequality," Tamkang Journal of Mathematics, vol. 28, no. 1, pp. 33-37, 1997.

[8] G. S. Yang and K. L. Tseng, "On certain integral inequalities related to Hermite-Hadamard inequalities," Journal of Mathematical Analysis and Applications, vol. 239, no. 1, pp. 180-187, 1999.

[9] İ. İşcan, "Hermite-Hadamard and Simpson-Like type inequalities for differentiable harmonically convex functions," Journal of Mathematics, vol. 2014, Article ID 346305, 10 pages, 2014.

[10] İ. İşcan and S. Wu, "Hermite-Hadamard type inequalities for harmonically convex functions via fractional integrals," Applied Mathematics and Computation, vol. 238, pp. 237-244, 2014. 


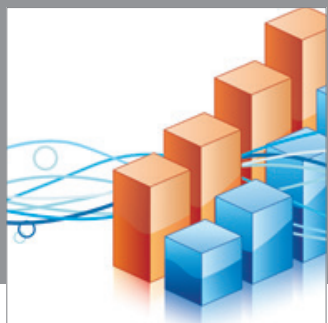

Advances in

Operations Research

mansans

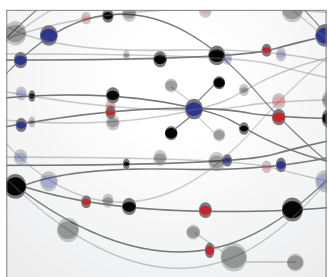

The Scientific World Journal
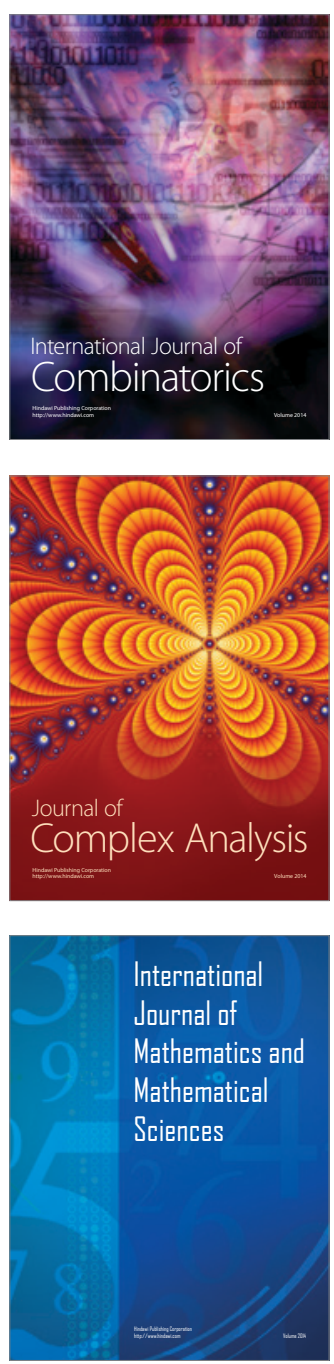
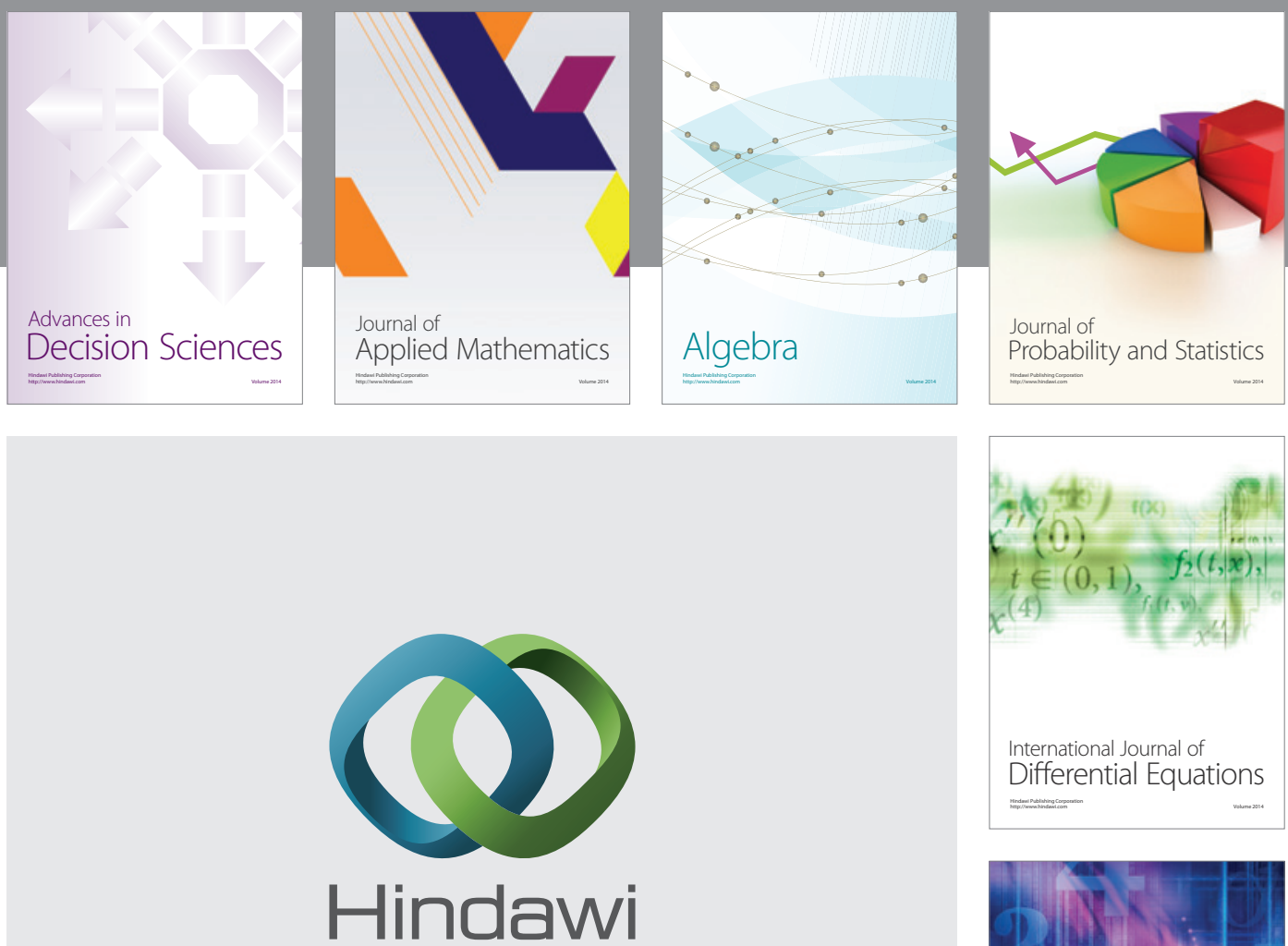

Submit your manuscripts at http://www.hindawi.com
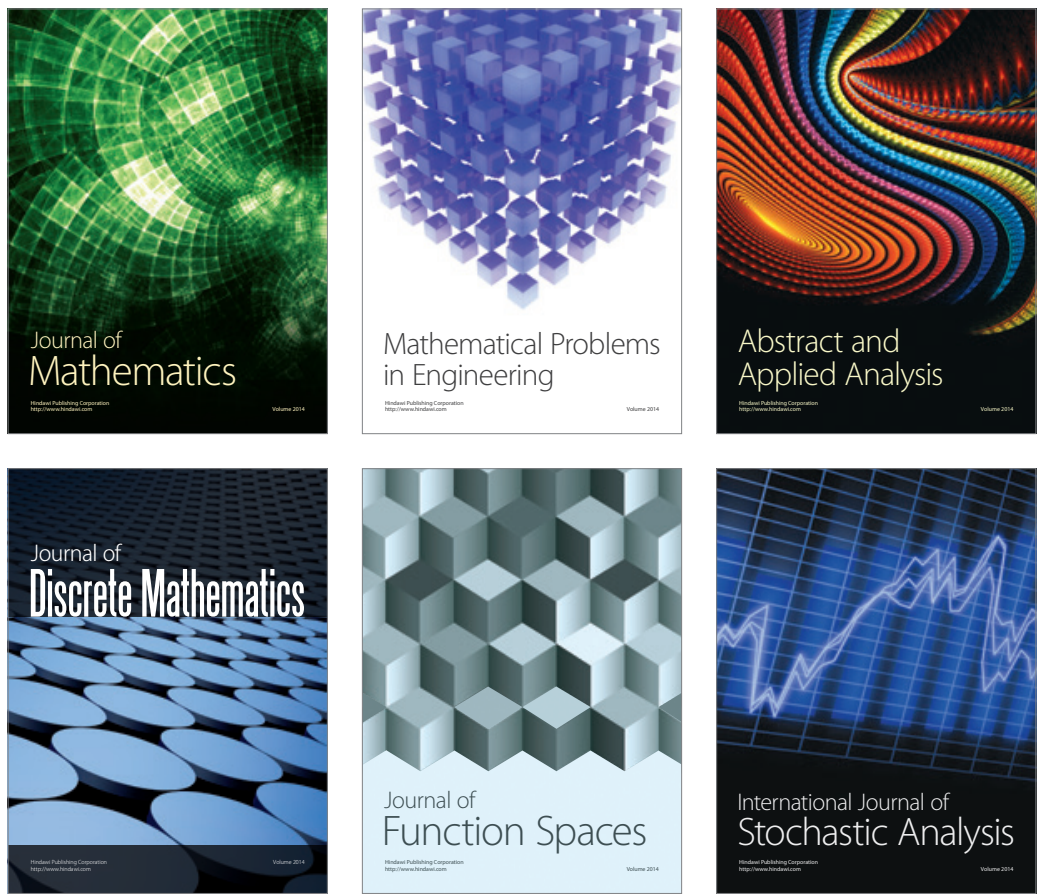

Journal of

Function Spaces

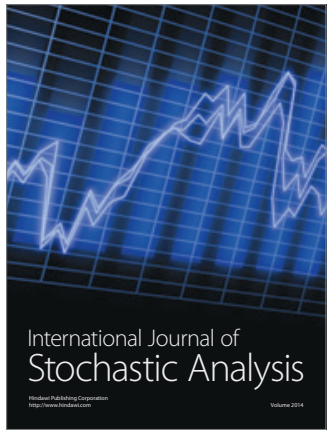

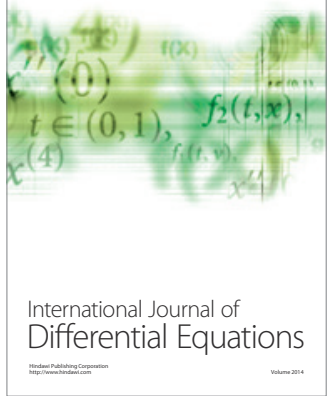
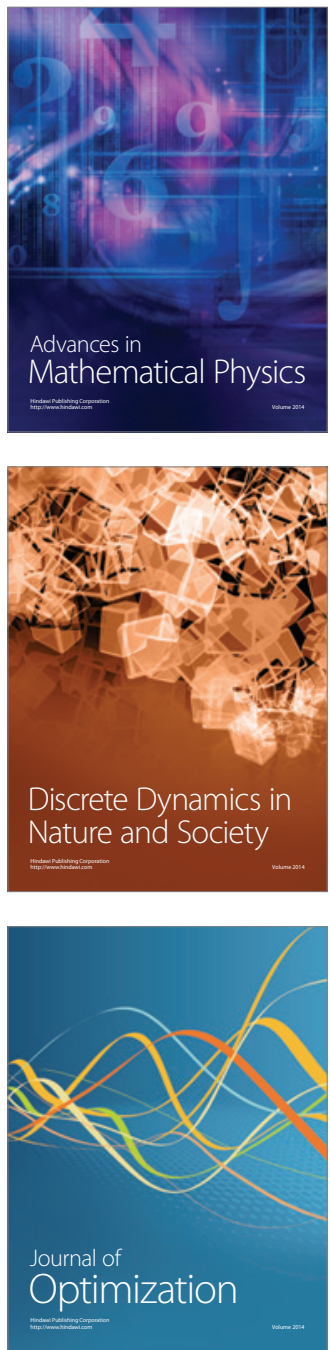